\title{
A response to high-dose estrogen for tamoxifen, aromatase inhibitor and trastuzumab refractory HER2+ and ER+ advanced breast cancer
}

\author{
B. Nervi, A. Denes, M. J. Ellis \\ Department of Medicine, Section of Medical Oncology, Washington University School of Medicine, \\ St Louis, MO, USA.
}

\section{Presentation and diagnosis}

A 66-year-old Caucasian woman without a significant past medical history underwent a routine bilateral mammography in October 2001 that showed a speculated mass in the right breast. Ultrasound examination of the right breast revealed two separate masses $(1.7$ and $1.4 \mathrm{~cm})$ suggestive of malignancy. A core biopsy showed invasive ductal carcinoma, and the patient underwent a right lumpectomy with sentinel lymph node dissection that revealed two separated invasive carcinomas: $1.5 \times$ 1.5 and $1.7 \times 1.3 \mathrm{~cm}$, poorly differentiated with signet ring features, extensive lymphovascular invasion, positive margins in one of the lesions, areas of in-situ lobular carcinoma, ER+, PR+, and HER2+ with gene amplification by FISH (Ratio of 5.6 for HER2 amplification adjusted for chromosome 17 polysomy; ratio of greater of 2 indicates amplification). One of fifteen axillary lymph nodes was positive for carcinoma with $a<0.2 \mathrm{~cm}$ metastatic implant. Subsequently the patient had a right modified mastectomy with TRAM reconstruction with no evidence of residual carcinoma in the breast, and one in two additional lymph nodes examined were positive for carcinoma. The tumor marker Ca 15-3 was elevated up to 85 units $/ \mathrm{ml}$ (normal $<32$ units $/ \mathrm{ml}$ ). She was found on staging evaluation (computerized

Correspondence to: Matthew J. Ellis, Washington University School of Medicine, 660 S Euclid Ave, Campus Box 8056, St Louis, MO 63110, USA. E-mail: mellis@wustl.edu; Tel: +314 362 3774; Fax: +314 3627096

Received: 09/08/05

Revised: 12/01/05

Accepted: 06/10/05

First published online 03/01/06

BCO/415/2005/CS tomographical (CT) scan, bone scan) to have radiographic findings consistent with mixed lytic-sclerotic metastasis in the left proximal femur. Follow-up evaluation with positron emission tomographical (PET) scan confirmed intense increase in uptake of $\mathrm{F}-18$ fluorodeoxy-glucose (FDG) within the left femoral intertrochanteric region, as well as a focus of increased activity in the right ischial region and the right iliac crest suspicious for bone metastasis.

In summary, this is a post-menopausal patient who presented with Stage IV breast carcinoma with bone metastasis and increased tumor marker $\mathrm{Ca}$ 15-3. The tumor biomarkers suggested that the tumor was estrogen dependent but amplification of HER2 was also present. Besides a mild left hip pain, the patient was asymptomatic with a performance status ECOG 0.

\section{Treatment and outcome}

The patient was treated initially with radiation to the left hip to prevent a bone fracture, biphosphonates to prevent skeletal complications associated with bone metastasis, and endocrine therapy with an aromatase inhibitor. The patient received zoledronic acid and exemestane with decrease in the tumor marker $\mathrm{Ca}$ 15-3 and stabilization in the progression of the disease for 7 months (Figure 1). The patient subsequently experienced progression in bone and she received fulvestrant, a pure estrogen receptor antagonist, and another aromatase inhibitor, letrozole for 5 and 2 months respectively, with progression of bone disease and significant elevation in the Ca 15-3 tumor marker up to 520 units $/ \mathrm{ml}$. At this point the patient still had a good performance status, 


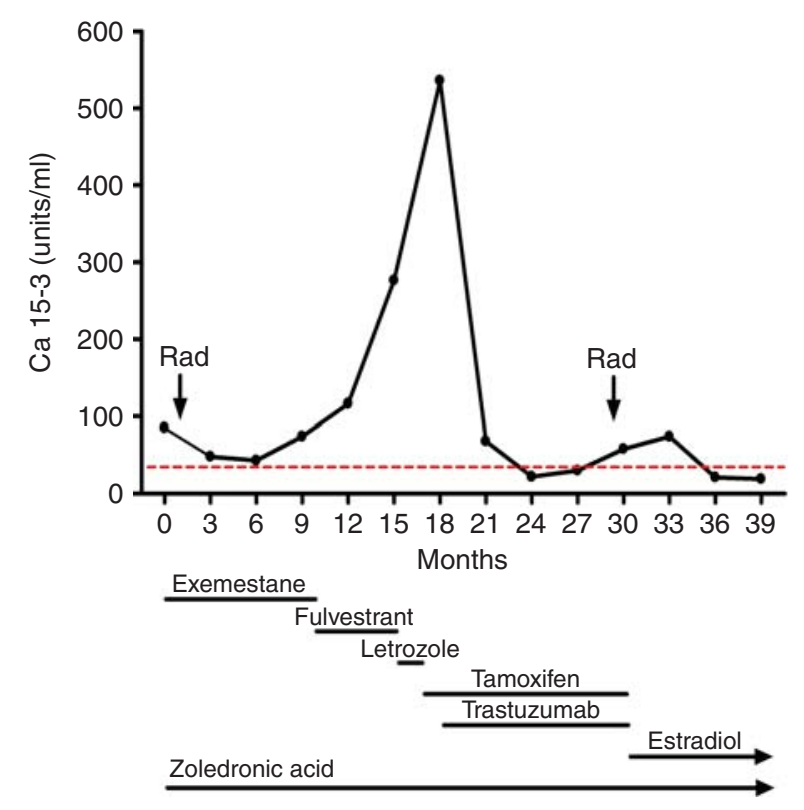

Figure 1.

Changes in Ca 15-3 tumor marker during different treatments. Dotted line marks the upper normal limit for Ca 15-3. In the bottom part is represented the course treatments the patient has received. Rad: palliative radiation therapy.

without bone pain. Subsequently, the patient received tamoxifen $20 \mathrm{mg}$ every day and trastuzumab every 3 weeks. The patient had a dramatic response with normalization in the tumor marker and stabilization in the progression of her bone metastatic disease. This combination was very well tolerated, had no significant side effects, and was effective for 9 months when the tumor showed progression again. The patient then experienced right hip pain and the bone scan showed an interval progression in the metastatic disease in the left femoral neck that correlated with CT findings of a sclerotic lesion in the same place. There were other lesions in the sacrum, right acetabulum, right iliac wing, and vertebral bodies of L5 and S1. The patient received radiation to the right hip to prevent an impending fracture with relief in the hip pain.

The patient was informed that the typical management approach following failure of multiple lines of endocrine treatment and trastuzumab would be to continue the trastuzumab and initiate chemotherapy. We also suggested the alternative of fifth-line endocrine therapy with high-dose estradiol, $10 \mathrm{mg}$ every 8 hours, and the patient took that option, based in quality of life considerations with the outcome indicated in Figure 1. Interestingly, estradiol treatment rapidly controlled disease progression with normalization in the tumor marker $\mathrm{Ca}$ 15-3, and after 9 months of treatment the patient is asymptomatic with stable osseous metastatic disease followed with bone and CT scans. The patient continues receiving monthly zoledronic acid and her yearly mammograms have been normal.

\section{Comments}

Remarkably, this patient with advanced ER+, HER2 + breast cancer, refractory to standard hormonal therapies (steroidal and non-steroidal aromatase inhibitors, fulvestrant, and tamoxifen), progressing following trastuzumab treatment in combination with tamoxifen, responded to estradiol therapy as fifth-line treatment without significant side effects. Although the mechanism responsible for efficacy of estrogen therapy is not completely understood, high doses of estrogen are thought to function principally by activating apoptotic pathways. Estradiol, which initially enhanced cell growth and tumor cell survival, appears to collapse the enhanced survival systems associated with endocrine-therapy-resistant disease progression [1]. Our case suggests that this event may not be impeded by constitutive HER2 signaling, opening the possibility of estradiol therapy in the setting of ER+ HER2 FISH+ disease, even after trastuzumab treatment.

The paradoxical anti-tumor action of high doses of synthetic estrogens in the treatment of breast cancer has a long history, and has been known since the 1950s [2]. In the 1970s, Ingle and colleagues showed in a randomized clinical trial of diethylstilbestrol (DES) vs. tamoxifen in post-menopausal women with advanced breast cancer $(n=143)$, similar response rates $(41 \%$ and $33 \%$ complete plus partial response for DES or tamoxifen respectively, $P=N S$ ) and similar progression free survival (142 vs. 171 days for DES or tamoxifen respectively, $P=$ NS) [3]. Toxicity was greater in patients receiving DES, with $9 / 74$ patients $(12 \%)$ discontinuing therapy solely because of adverse reactions. Since there was no statistically significant difference in efficacy and since tamoxifen was less toxic, tamoxifen was proposed as the preferred agent and as the standard of care endocrine therapy for breast cancer since the 1970s. However longer-term follow-up this trial suggested an overall survival advantage for the DES arm renewing interest in high-dose estrogen therapy, particularly as third or fourth-line endocrine treatment.

Whereas most of the data with high-dose estrogen is from patients receiving DES as first-line therapy for advanced disease, patients with previous exposure to multiple lines of contemporary endocrine therapy may be hyper-sensitized to estrogen therapy. A Phase II study of DES (5 mg, three times daily) following exhaustive anti-estrogen and aromatase inhibitor therapy showed $4 / 32$ complete responses, $6 / 32$ partial responses, and 2/32 stable disease [4]. 
However, the use of high-dose estrogen therapy is associated with breast tenderness, vaginal discharge, and, more seriously, congestive heart failure, venous thrombosis and malignant hypercalcemia and so there remains considerable reluctance to use this potentially valuable endocrine approach. However with modern supportive care and careful case selection these problems are manageable.

Resistance to SERMs, fulvestrant, and aromatase inhibitors, results from a complex series of changes that prevent apoptosis and thus enhance cell proliferation and survival. Alterations in several signal transduction pathways have been described for tamoxifen resistance, including enhanced activity of Activating Protein 1 (AP1) and phosphatidylinositol 3-kinase/protein kinase B (PKB or AKT) pathways and altered expression of protein kinase $\mathrm{C} \alpha$, HER2, and insulin-like growth factor signaling. All of these events have been linked to the activation of nuclear factor $\kappa B(N F-\kappa B)$.

$N F-\kappa B$ is a key transcriptional factor for cell growth and survival. Increased NF- $\mathrm{BB}$ activity is involved in drug resistance in breast cancer, and $\mathrm{ER} \alpha$ represses $\mathrm{NF}-\kappa \mathrm{B}$ activity in the presence of estradiol. Estradiol binds to ER $\alpha$, and the estradiol-ER $\alpha$ complex suppresses NF- $\mathrm{KB}$ activity by inhibiting the binding of

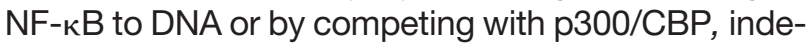
pendent of new protein synthesis. The decreased $\mathrm{NF}-\kappa \mathrm{B}$ activity induced by estradiol should increase the number of cells undergoing apoptosis [1].

Song and colleagues provided an important clue that it may be the Fas/FasL system which induces apoptosis in response to estrogen. They observed that in some estrogen deprived ER-positive breast cancer cells, apoptosis occurs with low estrogen concentrations, by enhancing activation of the Fas receptor [5]. This hypothesis has been extended to tamoxifen and raloxifene-stimulated breast cancer cells in vivo and in vitro [4]. Simultaneously, estradiol initiates the synthesis of the Fas receptor and the activation of caspase-8 from procaspase- 8 to induce apoptosis. The result is that tumors previously exposed to exhaustive anti-estrogen therapies rapidly regress in vivo in response to low physiologic concentrations of estradiol $(83-96 \mathrm{pg} / \mathrm{ml})$ within the post-menopausal range. In addition, high doses of estrogens reduce plasma levels of insulin growth factor (IGF), which act in concert to promote the survival of breast cancer cells [6]. Preclinical models also implicate a role for IGF-2 in response to highdose estrogen [7].

High-dose estrogens should be considered only in post-menopausal breast cancer patients because this option is ineffective before menopause [2]. DES is difficult to obtain in the USA, and a comparable choice is estradiol, $2 \mathrm{mg}$ tablets. The recommended dose is $30 \mathrm{mg} /$ day in fractionated doses. Important questions that remain are if it is possible to achieve the same clinical results with lower estrogen doses, and if the response to therapeutic estrogen reactivates response to anti-estrogen therapy, so treatments can be cycled. We are currently conducting a randomized Phase 2 trial of $6 \mathrm{mg}$ vs. $30 \mathrm{mg}$ of estradiol for women with advanced breast cancer refractory to aromatase inhibitors. As a consequence of our experience in this case we are amending the protocol to allow patients who have received trastuzumab in combination with either an aromatase inhibitor or tamoxifen.

\section{References}

1. Liu H, Chen J, Wang L, et al. Apoptotic action of 17betaestradiol in raloxifene-resistant MCF-7 cells in vitro and in vivo. J Natl Cancer Inst 2003; 95: 1586-1597.

2. Kennedy BJ. Hormone therapy for advanced breast cancer. Cancer 1965; 18: 1551-1557.

3. Ingle JN, Ahmann DL, Green SJ, et al. Randomized clinical trial of diethylstilbestrol vs. tamoxifen in postmenopausal women with advanced breast cancer. N Engl J Med 1981; 304: 16-21.

4. Lonning PE, Taylor PD, Anker G, et al. High-dose estrogen treatment in postmenopausal breast cancer patients heavily exposed to endocrine therapy. Breast Cancer Res Treat 2001; 67: 111-116.

5. Song RX, Mor G, Naflolin F, et al. Effect of long-term estrogen deprivation on apoptotic responses of breast cancer cells to 17beta-estradiol. J Natl Cancer Inst 2001; 93: 1714-1723.

6. Helle SI, Geisler J, Anger GB, et al. Alterations in the insulin-like growth factor system during treatment with diethylstilboestrol in patients with metastatic breast cancer. Br J Cancer 2001; 85: 147-151.

7. Brunner N, Spang-Thomsen M, Cullen K. The T61 human breast cancer xenograft: an experimental model of estrogen therapy of breast cancer. Breast Cancer Res Treat 1996; 39: 87-92. 